Article

\title{
Impulse Magnetization of Nd-Fe-B Sintered Magnets for Sensors
}

\author{
Marek Przybylski ${ }^{1, *}$, Dariusz Kapelski ${ }^{1}$, Barbara Ślusarek ${ }^{1}$ and Sławomir Wiak ${ }^{2}$ \\ 1 Tele \& Radio Research Institute, Ratuszowa 11 St, 03-450 Warsaw, Poland; dariusz.kapelski@itr.org.pl (D.K.); \\ barbara.slusarek@itr.org.pl (B.Ś.) \\ 2 Lodz University of Technology, Institute of Mechatronics and Information Systems, \\ Stefanowskiego 18/22 St, 90-924 Lodz, Poland; swiak@wp.pl \\ * Correspondence: marek.przybylski@itr.org.pl; Tel.: +48-22-590-7362
}

Academic Editor: Andreas Hütten

Received: 8 January 2016; Accepted: 12 April 2016; Published: 21 April 2016

\begin{abstract}
Magnetization of large Nd-Fe-B sintered permanent magnets is still challenging. This type of permanent magnet is electrically conductive, so impulse magnetization causes a flow of eddy currents which prevent magnetization of the whole volume of the magnet. The paper deals with the impulse magnetization of sintered Nd-Fe-B permanent magnets and shows a method for the determination of suitable parameters for the supply system. The necessary magnetic field strength for magnetization of the magnet to saturation was determined. The optimal magnetizing fixture supply voltage for magnetization to saturation was determined from simulations in PSpice software, finite element analyses in Maxwell 15 and measurements. Measurements of magnetic induction on the surface of the Nd-Fe-B magnet are also presented to ensure that a magnet with $70 \mathrm{~mm}$ diameter and $20 \mathrm{~mm}$ in height is fully saturated.
\end{abstract}

Keywords: Nd-Fe-B permanent magnets; permanent magnets; impulse magnetization; magnetic saturation; magnetic induction; coercivity

\section{Introduction}

Sintered Nd-Fe-B permanent magnets make it possible to increase the performance of devices and can also enable their miniaturization. The main field of application for this type of permanent magnet are electric machines such as electric motors and generators. For example, in direct current motors permanent magnets are applied in stators as an excitation source of magnetic field. In brushless direct current motors permanent magnets are applied to rotors. In small wind power plants permanent magnets are used in 3-phase axial flux machines, without a soft magnetic core, and with power of about a few $\mathrm{kW}$. With the development of magnetic materials the range of their application is broadening, among others in magnetic sensors. Developments in material engineering allow the elaboration of new materials with improved parameters and their application in a new generation of magnetic sensors. For many applications the diversity of the physical properties of the materials and the possibility of producing magnets with a complicated shape with two or more magnetic poles are very important and enable the application of magnets in magnetic sensors. These types of sensor are elements of many measuring devices. The main role of magnetic sensors is not only in the measurement of the distribution and parameters of the magnetic field, but also in the measurement of another physical quantities such as magnetic force, where the magnetic field transforms into a processing measurement signal. For many applications it is very important to magnetize to saturation the whole volume of the magnet and ensure a suitable distribution of magnetic induction on the surface of the permanent magnets in magnetic sensors [1,2]. 
Magnetic sensors with permanent magnets are often applied in measuring transducers, such as devices for the measurement of linear or rotational speed, in tachometers, in reeds, vibrations and pressure meters, etc. Three pairs of permanent magnets are used in a three degrees of freedom displacement measurement system to create magnetic field in the air. This magnetic field is measured by Hall sensors and their signals give position information. In this type of device, permanent magnets must be magnetized uniformly for accurate position measurement [3]. In turn, a permanent magnet is used in a noncontact force measuring system and must also be magnetized uniformly. In the case of non-uniform magnetization, the force will be less than expected in the design stage of the sensor because a permanent magnet produces less magnetic flux [4]. Permanent magnets are also used in electromagnetic flow meters for liquid metals. The converter consists of a contactless electromagnetic pump with a torque sensor mounted on the pump shaft. The electromagnetic pump is composed of two rotating steel discs with embedded permanent magnets with alternating poles. The rotation of the discs creates a travelling sinusoidal magnetic field and eddy currents within the liquid metal. The interaction of the magnetic field and induced eddy currents generates electromagnetic Lorentz force that provides the pumping effect. The flow rate is proportional to this force, and the torque is measured by a torque sensor and is converted to a flow rate. In this meter, uniform magnetization is essential for trustworthy flow measurement of the liquid metal, especially for pipes with large diameters and thicknesses [5].

Sintered Nd-Fe-B magnets have high magnetic energy and this kind of permanent magnet is mainly magnetized in the last stage of production using high voltage (about $5 \mathrm{kV}$ ) impulse magnetizers equipped with capacitors with large capacitance [6]. The electric energy stored in the capacitors is discharged by a magnetizing fixture, creating the magnetic field needed for magnetization.

Each hard magnetic material has a defined magnetic field strength that enables its magnetization to saturation. Producers of permanent magnets do not often provide such information. If they do provide the value of this magnetic field strength, often it is more than practically needed. A larger magnetic field causes higher electric current impulses and the magnetization fixture is mechanically strained more than necessary. This can mechanically and thermally spoil the magnetization fixture earlier than expected.

Magnetic, electric, electromagnetic and electromechanical devices need permanent magnets with different numbers and configurations of magnetic poles. Larger magnets with two or more magnetic poles can be composed of two pole magnets. However, in this situation there are many problems, e.g., gluing of magnets, or imperfect distribution of magnetic flux density in the air gap. A magnetizing fixture should be designed and made for the required dimensions of the magnet, number of magnetic poles and their patterns. Sometimes a magnetizing fixture can be universal for some types of permanent magnets, such as two pole with axial magnetization. Permanent magnets with different dimensions can be magnetized in this type of fixture. The amount of electrical energy gathered in a bank of capacitors determines the size and weight of the permanent magnets to be magnetized.

$\mathrm{Nd}-\mathrm{Fe}-\mathrm{B}$ sintered permanent magnets are made of electrically conductive material. That is why in these kinds of magnet, during impulse magnetization, eddy currents flow and cause inhomogeneous magnetization, power loss and unwanted heating of the magnets. This can be mainly seen in magnets with a large volume.

Impulse magnetizers are built as low voltage (450-500 volts), medium voltage (800-1000 volts) and high voltage (1500-3000 volts) appliances. Higher voltage magnetizers produce shorter magnetic field impulses and are more efficient. A process of impulse magnetization of magnets using condensers was first applied in about 1944, but there was a problem with such magnetizers. They often produced, depending on the resistance of the magnetizing fixture, oscillating currents which in a second pulse causes demagnetization of the magnet [6]. Improved magnetizers were applied from 1954 [7]. Two-pole magnets can be magnetized axially or diametrally in the case of cylindrical and ring magnets. Permanent magnets can be magnetized before or after mounting in a device [8,9]. In the case of ring magnets, they can be magnetized radially, also called homopolarly (one pole on an internal diameter and the other on an external diameter) [10]. Multipolar magnets can be magnetized radially or axially. 
The magnetization of a bush magnet with four radial poles was analyzed in [11-14]. In turn, the magnetization of an axial eight-pole magnet was analyzed in [15,16], and six-pole in [17].

First, for an analysis of the impulse magnetization process, analytical calculations were used. Since the 1980s, with improved computers, numerical analysis has been introduced in the design of magnetizers and the features of impulse magnetization. Nakata and Takahashi performed the first analyses of a transient magnetic field in a capacitor-discharge impulse magnetizer. They combined Maxwell's equation with Kirchhoff's equation for an electromagnetic analysis of the magnetization of a four-pole polymer-bonded magnet [11]. However, they did not consider eddy currents in the magnet, but took into account the eddy currents in the pole piece and yoke of a magnetizing fixture. Lee in his article deals with the magnetization of an axially oriented eight-pole epoxy-bonded Nd-Fe-B magnet. This type of magnet has high resistivity and in these magnets eddy currents can be neglected. However, it was also assumed that the magnet has a permeability equal to 1 and this was represented as an air gap in simulations [15]. This assumption is valid only for highly saturated permanent magnets. Jewell, Howe and Birch analyzed the magnetization of a four-pole radial Nd-Fe-B permanent magnet for motors [12]. In this research, they took eddy currents into consideration in magnetizing a bush magnet, but the magnet was only $1.5 \mathrm{~mm}$ thick and the eddy currents could also be neglected. A simulation of magnet magnetization was also conducted in-situ in the vicinity of soft magnetic materials, so the current required was not as high as in an air-core magnetizing fixture. Magnet magnetization in a mounted motor is difficult also because of the existence of bulk soft magnetic materials in a motor. The same authors conducted magnetization and measurements of electric parameters. Magnetization of a fully mounted motor is difficult and full saturation is hard to obtain because of the insufficient capacitance of the capacitors [13,14]. Heiden, Arkadan and Brauer also reported in [18] that magnetization of an Nd-Fe-B magnet, with the use of a magnetization fixture with a soft magnetic core, was not fully conducted due to eddy currents induced in the magnet. Air-cored magnetizing fixtures are easier to analyze because the inductance of the magnetizing winding is linear and an impulse current can be closely calculated. A four-pole radial field magnetizing fixture was applied for magnetization of an isotropic Nd-Fe-B bonded magnet. Because of the high resistivity of the magnet, impulse eddy currents in this magnet were neglected [19]. An axial field multipole magnetizing fixture was designed and used for magnetization of a six-pole magnet. This type of magnetizing fixture is air-cored and $24 \mathrm{kA}$ peak currents are produced [17]. Magnetization of an eight-pole planar Nd-Fe-B ring bonded magnet was analyzed and a magnetizing fixture for this magnet was designed and applied. This fixture uses soft magnetic composite (SMC) material for the magnetic core. Due to the high resistivity of the SMC material, it was possible to decrease the impulse magnetizing currents in comparison with air-cored fixtures. The SMC material enabled uniform magnetization of the core and the lack of displacement of magnetic flux from the magnetic core $[16,20]$. Multipolar magnetization of a planar and cylindrical permanent magnet with 36 magnetic poles resembling a chess-board was developed. These magnets could be applied in rotary linear actuators and planar electric motors [21,22]. Recently, for a description of the magnetization process of permanent magnets, the finite element method has been used with the Jiles-Atherton hysteresis model [23].

In the paper the magnetization process of an Nd-Fe-B permanent magnet is considered and optimized electrical supply parameters were determined for magnetization of the magnet practically to saturation. Impulse currents with low values enable electrical energy savings during magnetization of permanent magnets, especially in mass production. What is more, it is possible to increase the number of magnets magnetized in the same amount of time, because energizing the capacitors to a lower voltage takes less time.

\section{Materials and Methods}

The research was conducted on commercially available grade N38 anisotropic sintered Nd-Fe-B permanent magnets. These permanent magnets, according to supplier data, have the following parameters: remanence $B_{r}=1.25 \mathrm{~T}$, coercivity of magnetic flux density min. $\mathrm{H}_{\mathrm{Cb}}=899 \mathrm{kA} / \mathrm{m}$, coercivity 
of magnetic polarization min. $\mathrm{H}_{\mathrm{cJ}}=955 \mathrm{kA} / \mathrm{m}$, maximum value of $(\mathrm{BH})$ product $(\mathrm{BH})_{\max }=310 \mathrm{~kJ} / \mathrm{m}^{3}$, resistivity $\rho=1.44 \times 10^{-6} \Omega \cdot \mathrm{m}$ [24]. A permanent magnet $70 \mathrm{~mm}$ in diameter and $20 \mathrm{~mm}$ in height was chosen for the analysis of impulse magnetization.

For determination of the saturation magnetic field, samples made of N38 material with dimensions $30 \times 10 \times 10 \mathrm{~mm}$ with the direction of anisotropy according to $10 \mathrm{~mm}$ length were used. It was not possible to measure the parameters of the magnet $70 \mathrm{~mm}$ in diameter and $20 \mathrm{~mm}$ in height that was chosen for this study, due to the limitations of the measuring equipment. Measurements of magnetization and demagnetization curves were conducted using a hysteresisgraph AMH-20K-HS manufactured by Laboratorio Elettrofisico Walker LDJ Scientific (Nerviano, Italy). Measurements were conducted based on IEC standard IEC 60404-5.

Impulse magnetization was conducted using an impulse magnetizer. A magnetization fixture was supplied from an impulse magnetizer, designed and manufactured in the Tele \& Radio Research Institute, that consists of a bank of capacitors with $C=1 \mathrm{mF}$ capacitance and regulated voltage $U_{c}$ from 0 to $5000 \mathrm{~V}$. The impulse current is triggered by a thyristor as an electronic switch. The impulse magnetizer is also equipped with a diode that enables an aperiodical shape of current waveforms $v s$. time in the winding. The impulse magnetizer is supplied from an AC $230 \mathrm{~V}, 50 \mathrm{~Hz}$ line.

The magnetizing fixture was designed and manufactured by Wroclaw University of Technology. It is a cylinder with an internal diameter of $80 \mathrm{~mm}$ for a magnet to be magnetized and an external diameter of $150 \mathrm{~mm}$ and $193 \mathrm{~mm}$ in height. The magnetization fixture consists of an internal copper coil with 440 turns of $3 \mathrm{~mm}$ diameter wire and an external tube of aluminium with a diameter of $150 \mathrm{~mm}$ and $4 \mathrm{~mm}$ thickness to ensure high strength against high impulse currents and forces. The aluminium tube helps to increase the mechanical strength of the coil and allows easier emission of the heat that comes from the currents in the wires during frequent magnetization. This tube is paramagnetic and is sufficient for the mechanical safety of the coil. The coil and the tube have the same axis of rotation.

\section{Results}

\subsection{Determination of Saturation Magnetic Field}

Figure 1a shows the magnetization curves $B=f(H)$, magnetic flux density as a function of magnetic field strength and $J=f(H)$, and magnetic polarization as a function of the magnetic field strength for the maximum magnetizing field strength $\mathrm{H}_{\max }=1000 \mathrm{kA} / \mathrm{m}$. Measurements of the demagnetization curves are presented in Figures $1 \mathrm{~b}$ and 2.

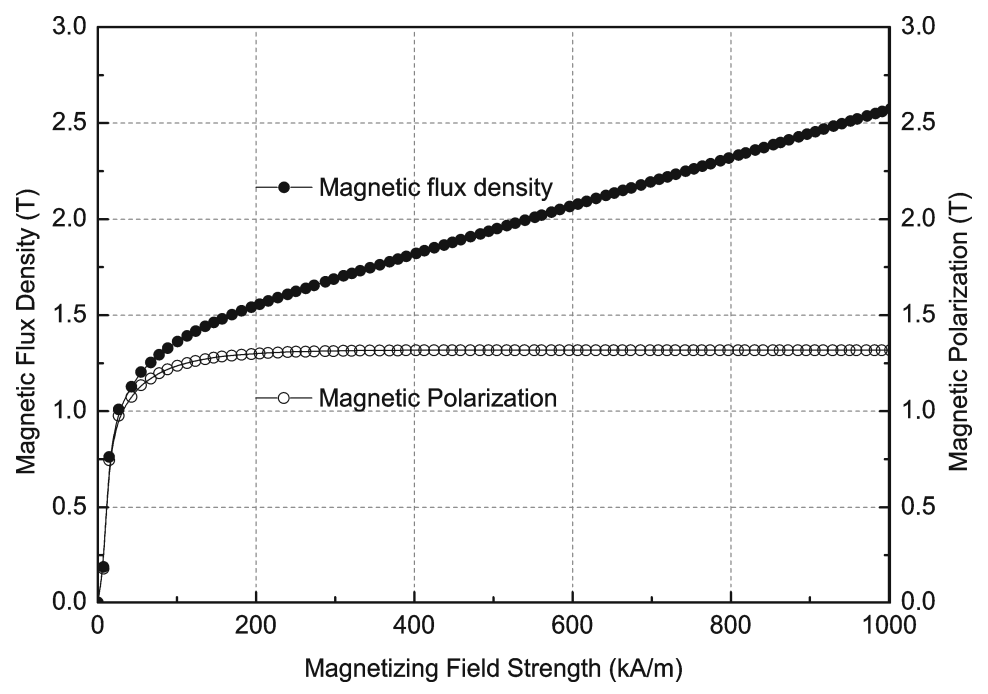

(a)

Figure 1. Cont. 


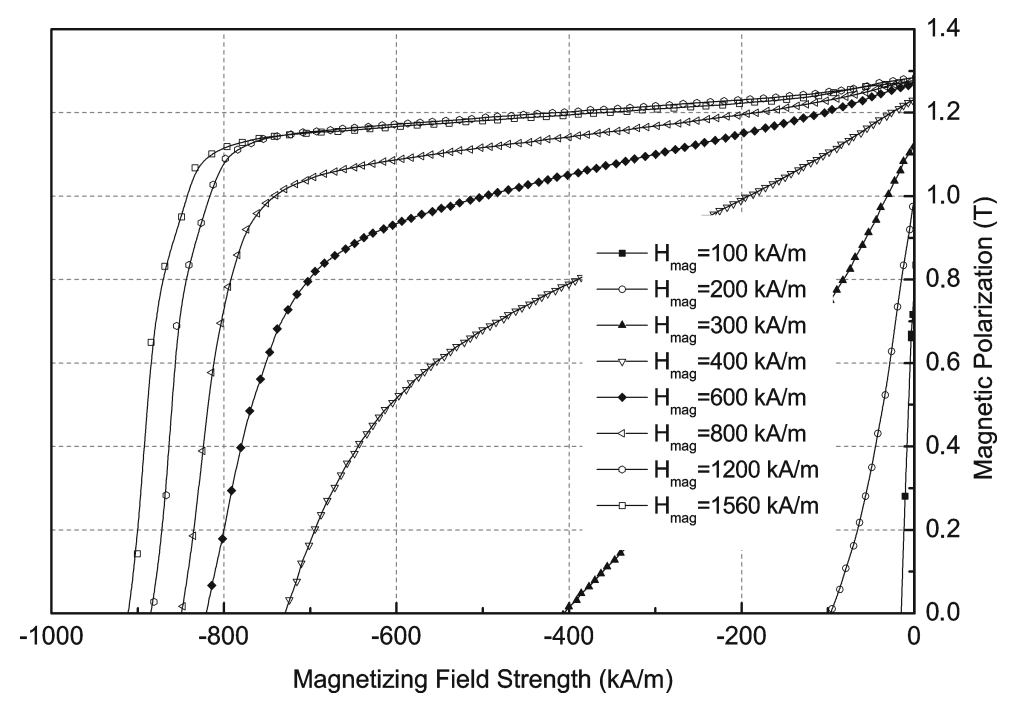

(b)

Figure 1. Measurements of (a) magnetic flux density as a function of magnetic field strength $B=f(H)$ and magnetic polarization as a function of magnetic field strength $J=f(H)$ magnetization curves; (b) $\mathrm{J}=\mathrm{f}(\mathrm{H})$ demagnetization curves of an Nd-Fe-B permanent magnet.

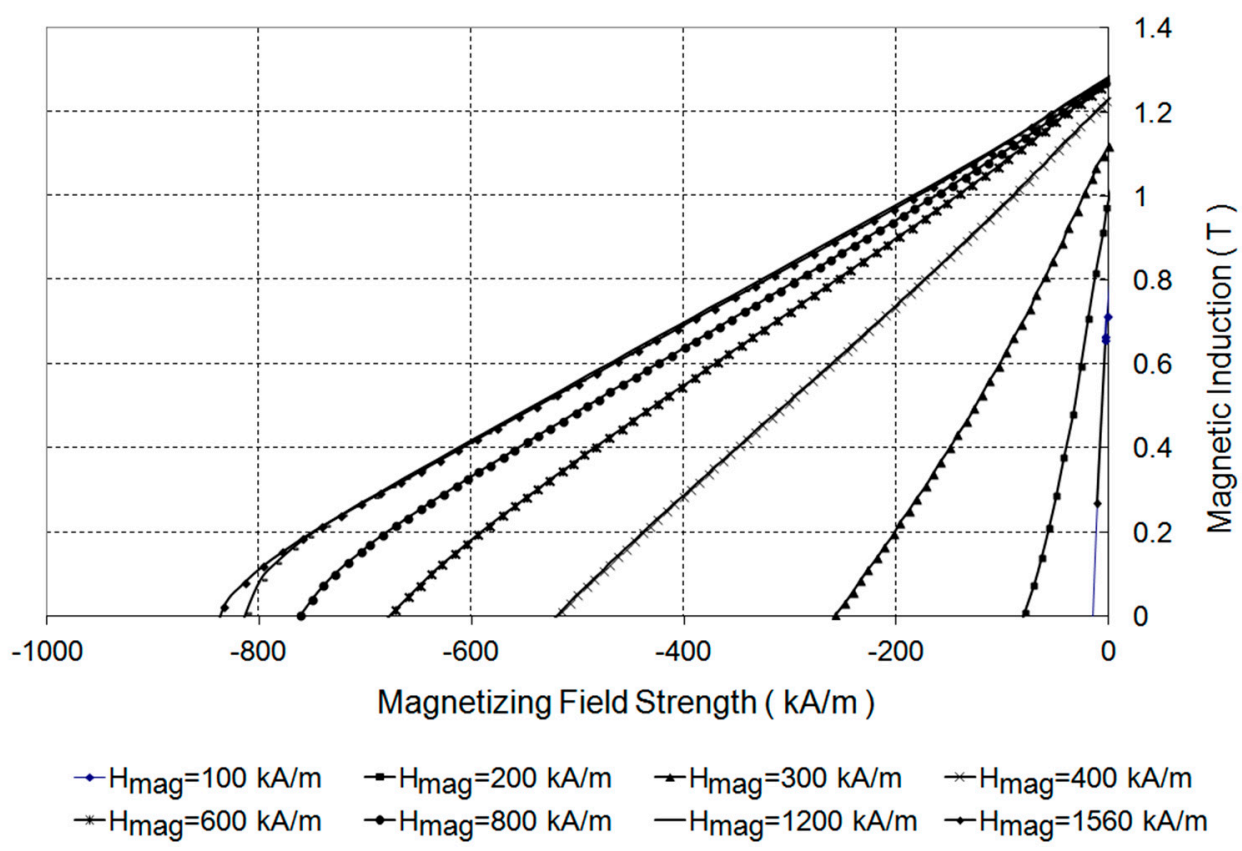

Figure 2. Measurements of $B=f(H)$ demagnetization curves of an Nd-Fe-B permanent magnet.

From the demagnetization curves it is possible to determine the parameters of permanent magnets such as remanence $B_{r}$, coercivities $H_{c B}, H_{c J}$ and maximum energy product $(B H)_{\max }$. Figure $1 b$ presents the demagnetization curves $\mathrm{J}=\mathrm{f}(\mathrm{H})$ for magnetic field strengths from the $\mathrm{H}_{\text {mag }}=100$ to $1560 \mathrm{kA} / \mathrm{m}$ range. The magnetization direction was parallel to the anisotropy direction. The permanent magnets were thermally demagnetized after each measurement cycle. Figure 2 shows the results of measurements of the second quadrant of the hysteresis loop of an Nd-Fe-B permanent magnet with different magnetizing field strengths.

Figure 2 shows that the second quadrant's characteristic magnetic induction values as a function of the magnetic field strength of the magnet when magnetized with $1200 \mathrm{kA} / \mathrm{m}$ and more overlap, 
and this ensures the distribution of magnetic induction on a surface with the same values. This means that a magnetization field strength of $1200 \mathrm{kA} / \mathrm{m}$ is enough for magnetization to saturation.

Figure 3 shows the measurements of remanence and coercivity of a sintered Nd-Fe-B permanent magnet as a function of the magnetization field strength. The results show that the remanence for magnetizing fields greater than $600 \mathrm{kA} / \mathrm{m}$ does not increase. The coercivity of the samples increases with increasing magnetizing field strength, but from the value of magnetizing field strength of $600 \mathrm{kA} / \mathrm{m}$ increases only slowly. The coercivity, as shown in Figure 3b with increasing magnetization field from $1000 \mathrm{kA} / \mathrm{m}$ to $1600 \mathrm{kA} / \mathrm{m}$, increases only by about $4.7 \%$, while the increase of the magnetization field is about $60 \%$. When all the magnetization field vectors are parallel to the external magnetic field, then the magnet is magnetized to saturation.
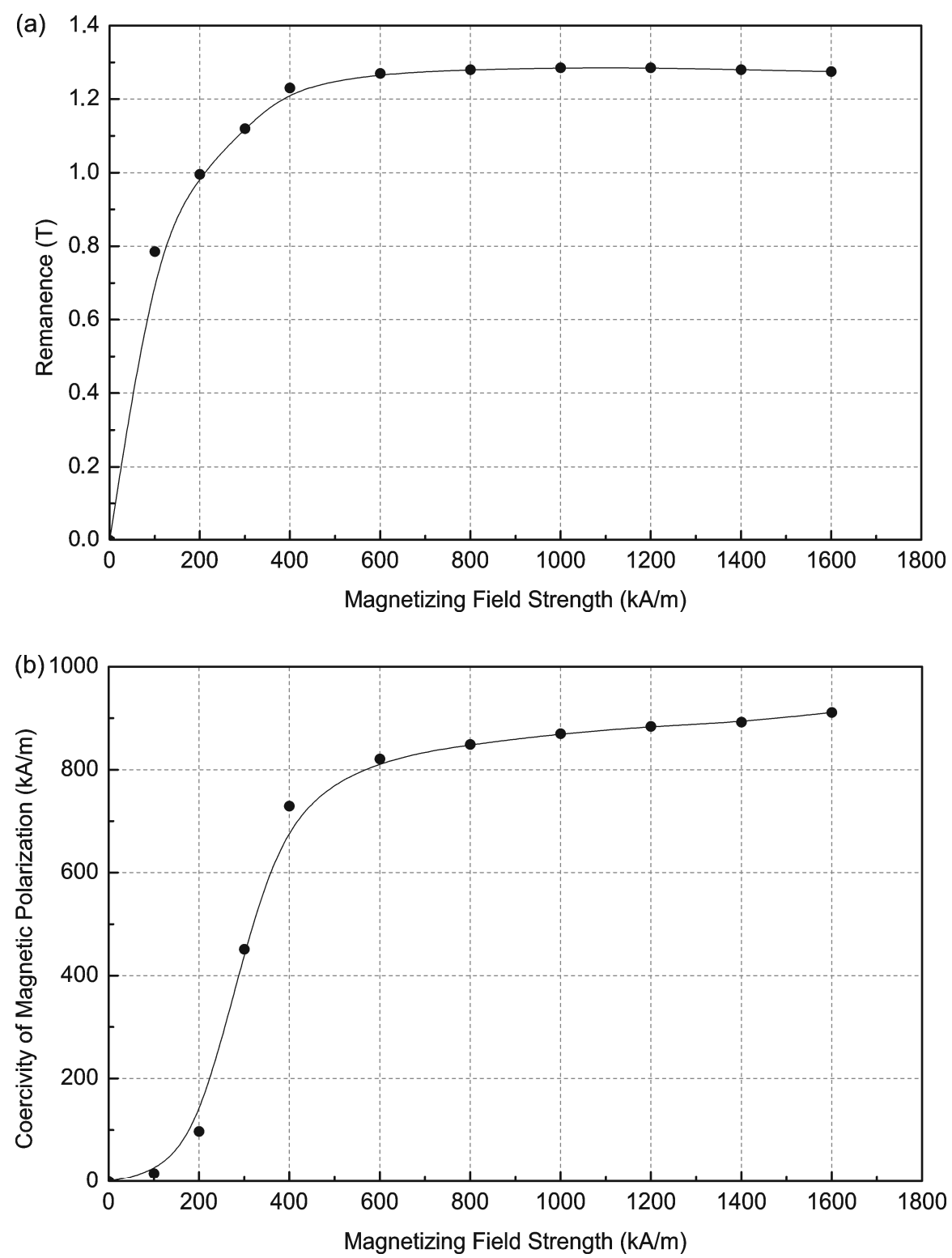

Figure 3. Measurements of (a) magnetic remanence $\mathrm{Br}$ and (b) coercivity of magnetic polarization $\mathrm{H}_{\mathrm{cJ}}$ as a function of magnetizing magnetic field strength $\mathrm{H}_{\text {mag }}$. 


\subsection{Impulse Magnetizer and Magnetization Fixture}

The analysis of the impulse magnetization process of permanent magnets made of sintered $\mathrm{Nd}-\mathrm{Fe}-\mathrm{B}$ alloys was conducted in several steps. These steps led to the determination of the supply parameters that enable magnetization of the magnet to saturation in the whole volume, not only on the surface. The first step was to calculate the magnetization fixture's electrical parameters from the dimensions and materials of the fixture. Because of the existence of the aluminium tube, the magnetizing fixture must be considered as a coreless transformer with a short-circuited secondary winding and cannot be treated as an air coil. This presents some difficulties in the analysis of the current in the magnetizing fixture's coil. What is more, eddy currents induced in the aluminium tube cause an opposite magnetic field which decreases the magnetic field inside the magnetizing fixture. Figure 4 presents an electric diagram of the magnetizer with a magnetizing fixture.

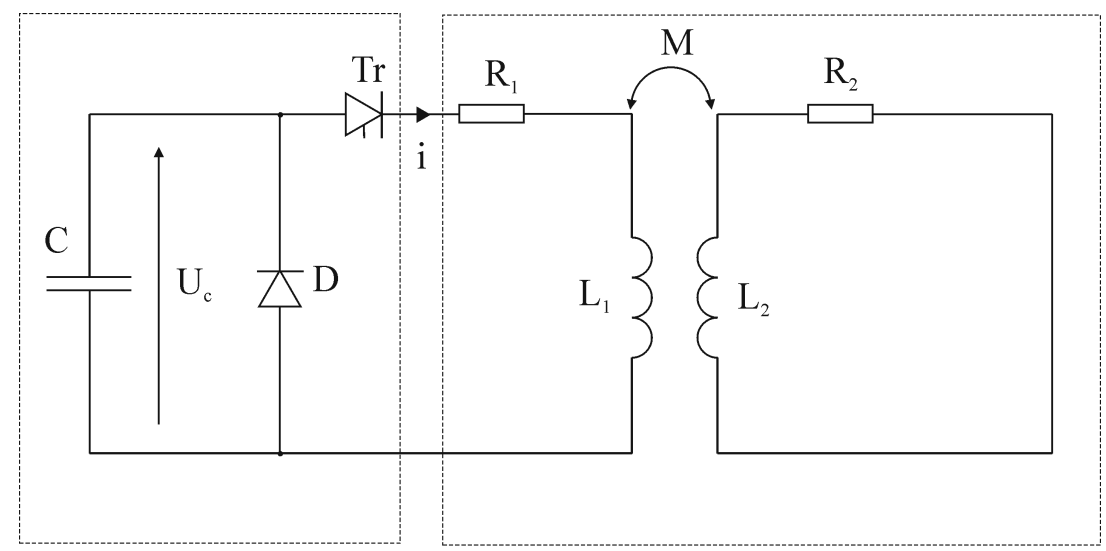

Impulse magnetizer

Magnetizing fixture

Figure 4. Electric diagram of the impulse magnetizer with a magnetizing fixture.

$R_{1}$ and $L_{1}$ represent the resistance and self-inductance of the coil. $R_{2}$ and $L_{2}$ represent the resistance and self-inductance of the aluminium tube. The magnetic coupling between the coil and aluminium tube is represented by mutual inductance $M$. The resistance of the coil was measured and $\mathrm{R}_{1}=0.38 \Omega$. Values of self, mutual inductances and resistance of the aluminium tube were calculated from the dimensions and number of turns of the magnetizing fixture and are equal to $\mathrm{L}_{1}=5.52 \mathrm{mH}$, $\mathrm{R}_{2}=17.10 \mu \Omega, \mathrm{L}_{2}=73.22 \mathrm{nH}, \mathrm{M}=11.40 \mu \mathrm{H}$. These parameters were essential for subsequent analyses with PSpice software.

\subsection{Analysis of Impulse Magnetization of Nd-Fe-B Magnet}

Next a series of numerical calculations were conducted in PSpice software, which is an electric circuit simulator that uses the electrical parameters of elements, such as resistance, inductance and capacitance. Measurements of currents in the coil for impulse magnetization were compared with PSpice calculations. As an example, Figure 5a shows the measurements of the impulse currents in the coil of the magnetization fixture. Measurements of current waveforms were conducted using a Tektronix TDS 210 oscilloscope (Beaverton, OR, USA) with a Tektronix A 621 AC current probe. Figure $5 b$ shows the comparison of the measurement and simulation of the magnetizing current for $\mathrm{U}_{\mathrm{c}}=3200 \mathrm{~V}$. The differences between the measurement curve and the simulation curve are caused by inaccuracy in determining parameters such as the resistance of the aluminium tube $\mathrm{R}_{2}$, inductances $\mathrm{L}_{1}$ and $\mathrm{L}_{2}$, and mutual inductance $\mathrm{M}$. Further inaccuracy is a result of the changing parameters of the capacity of capacitor $C$ with time, and the definition of thyristor $\mathrm{Tr}$ as an ideal element in PSpice software. These parameters were used in simulations in PSpice software. 


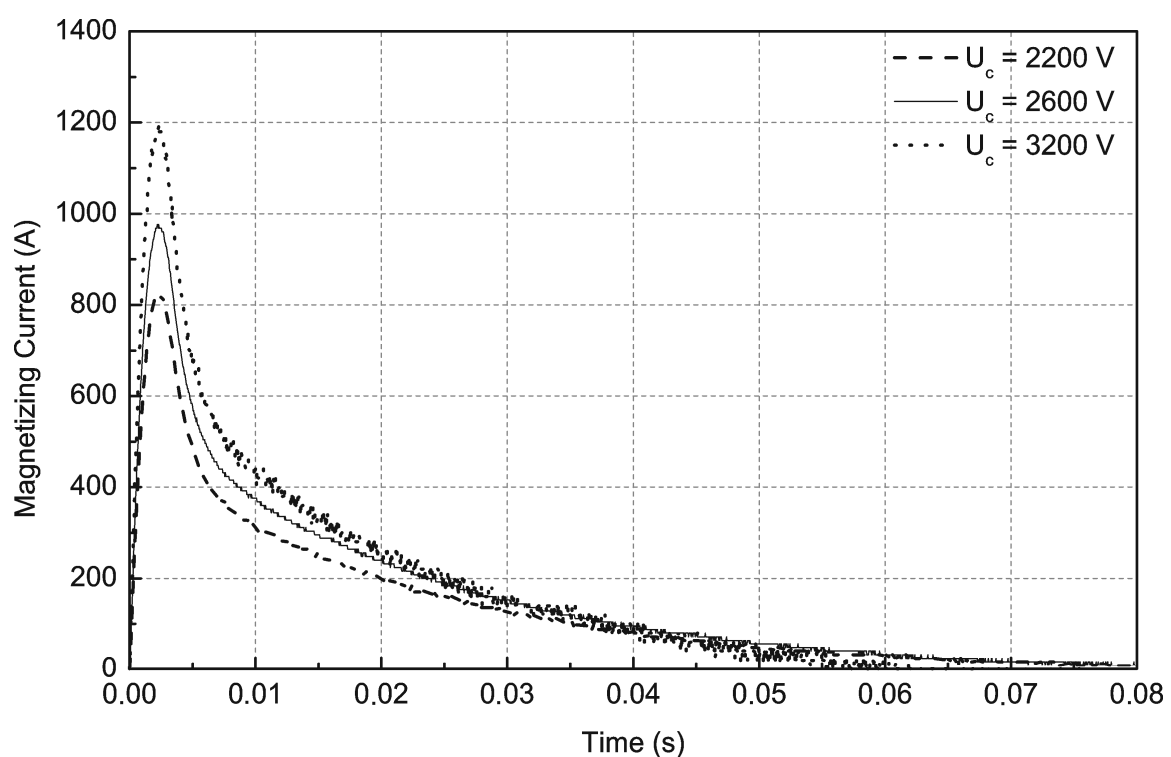

(a)

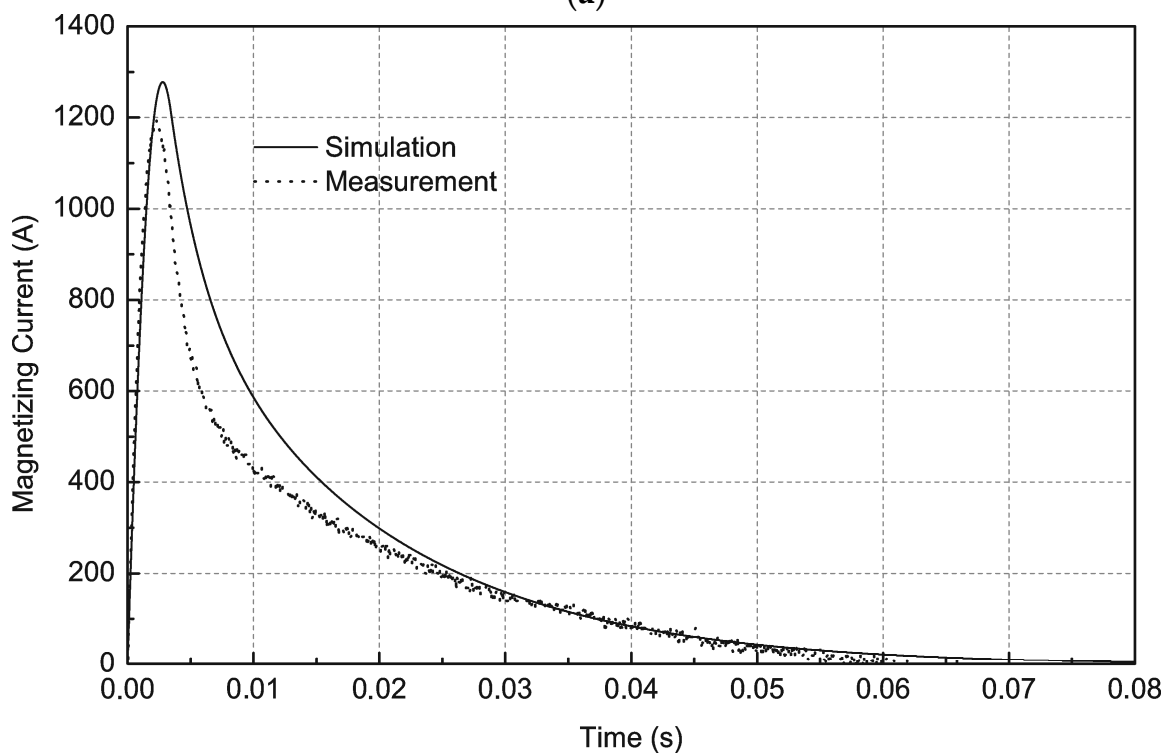

(b)

Figure 5. (a) Measurements of impulse magnetization currents for different voltages; (b) comparison of impulse magnetization currents for voltage $U_{c}=3200 \mathrm{~V}$.

The impulse magnetizer with capacitors charged to $3200 \mathrm{~V}$ creates in the magnetizing fixture an impulse current with a maximum $\mathrm{I}_{\mathrm{m}}=1200 \mathrm{~A}$ (Figure 6b). This current produces an impulse of magnetic flux density in the middle of the magnetizing fixture equal to $\mathrm{B}_{\mathrm{m}}=2.28 \mathrm{~T}$ (Figure 6a), measured by a fluxmeter with a THS 119 Hall sensor (Toshiba, Tokyo, Japan), with a supply circuit [25]. The curves in Figure 6 show that the magnetic induction increases more slowly than the current and decreases for longer than the current. This is caused by eddy currents induced in the aluminium shell of the coil. 
(a)

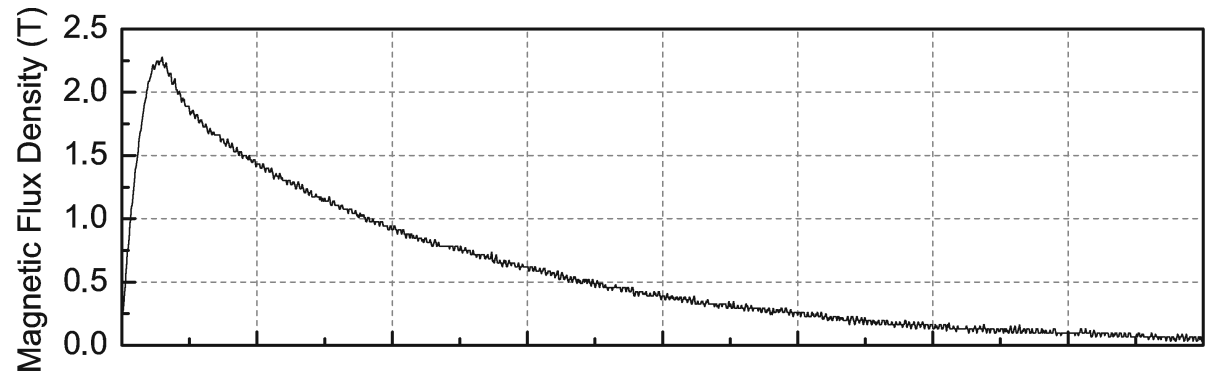

(b)

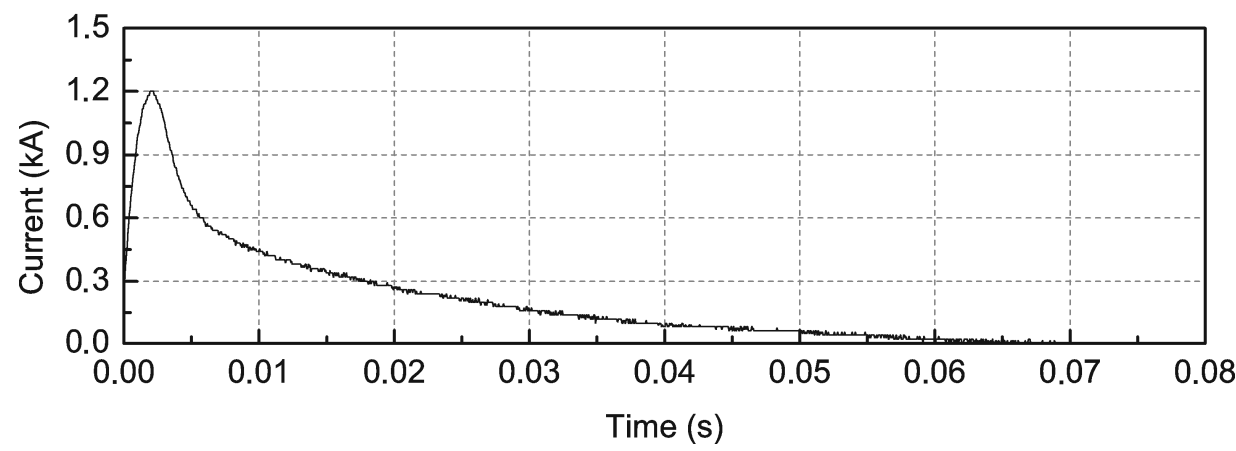

Figure 6. Measurements of (a) magnetic flux density in the middle of magnetization coil and (b) current, for $U_{c}=3200 \mathrm{~V}$ magnetization voltage as a function of time [25].

Computer calculations were performed for comparison in the Maxwell 15 finite elements method (FEM) software. The course of the current from upper Figure 6 was implemented in FEM software as the source of magnetic field. The simulated time course of magnetic induction in the middle of the coil is presented in Figure 7. Calculations were performed in two dimensions because the magnetizing fixture has axial symmetry. The maximum value of magnetic induction is equal to $2.56 \mathrm{~T}$. The course is not smooth because the current course implemented in FEM software is composed from only 20 points due to Maxwell 15 limits.

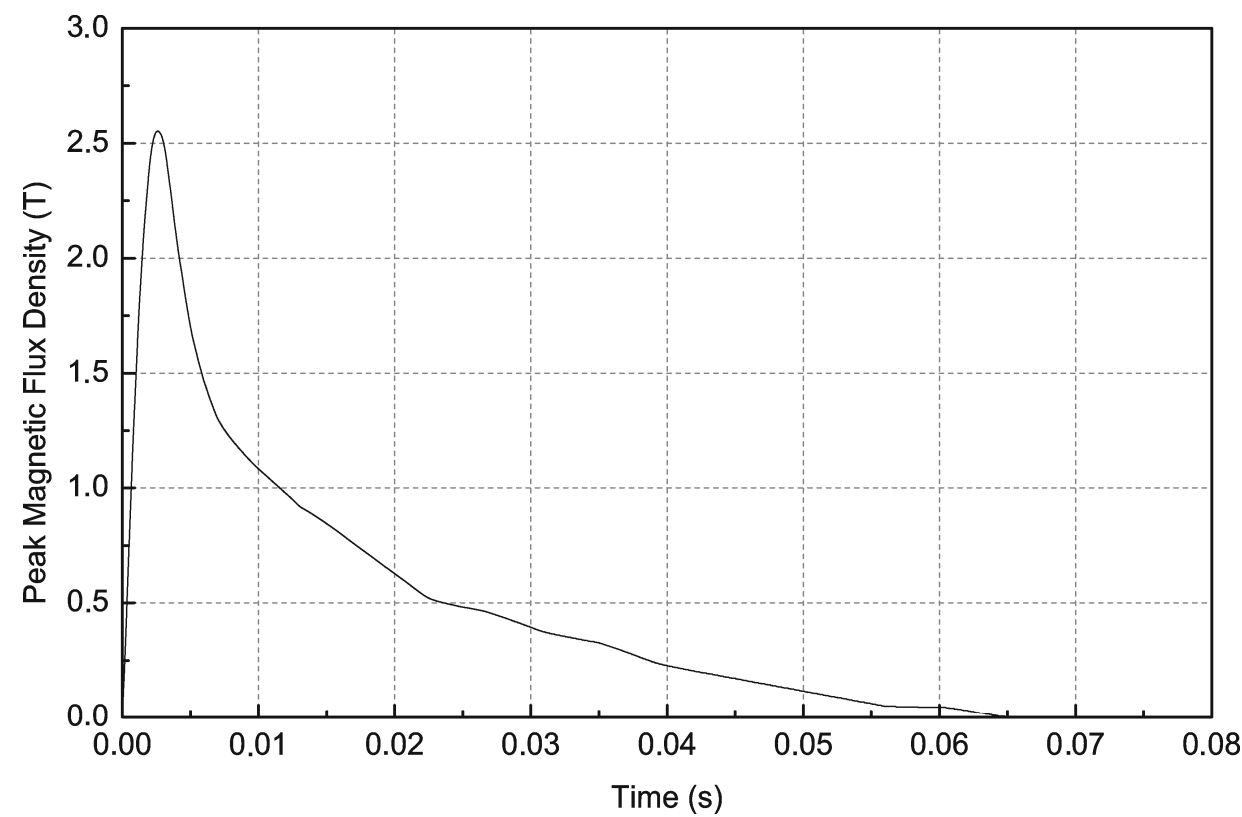

Figure 7. Simulation of magnetic flux density as a function of time in the middle of magnetizing coil in Maxwell 15. 
The difference between the measured and simulated maximum values of induction is about $12 \%$. This may be caused by numerical errors in the FEM software and measurement errors in the THS 119 Hall sensor fluxmeter.

The measured current characteristics $i(t)$ presented in Figure 5a, with different supply voltages, were exported to the FEM software as the supply currents. Numerical calculations of the magnetic flux density in the $70 \mathrm{~mm}$ diameter $20 \mathrm{~mm}$ high magnet were performed and are presented in Figure 8. The magnetic flux density in the centre of the magnet for $U_{c}=2800 \mathrm{~V}$ is equal to $2.60 \mathrm{~T}$, which is practically sufficient for magnetization to saturation according to Figure $3 a, b$.

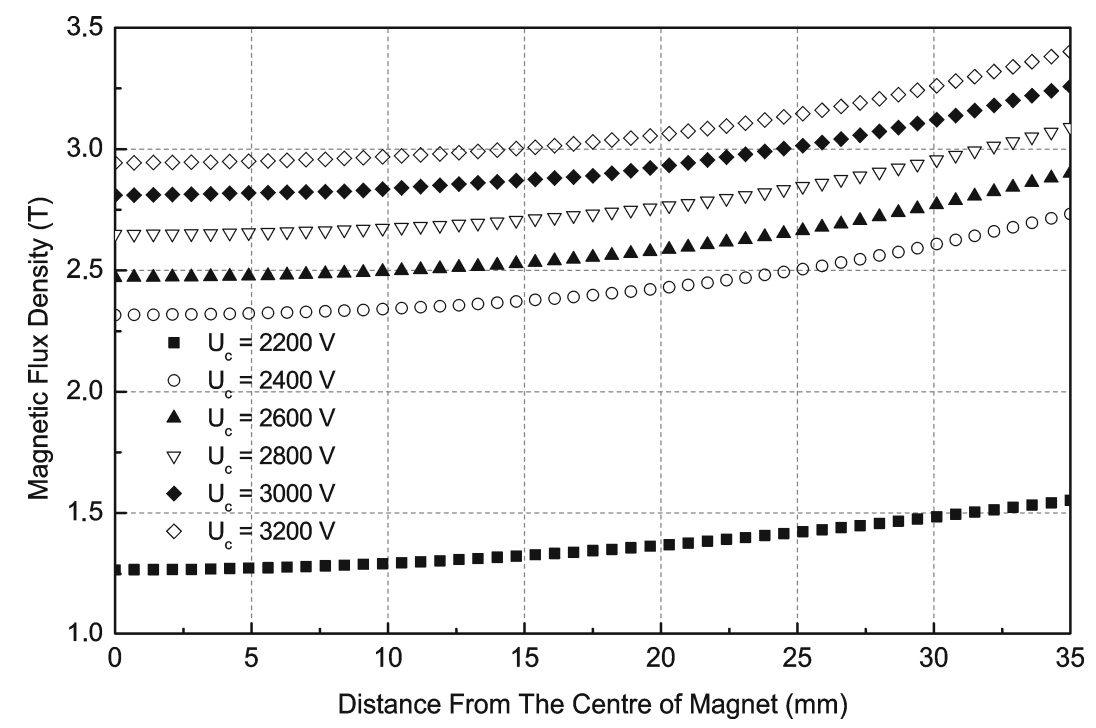

Figure 8. FEM simulations of magnetic induction in the Nd-Fe-B sintered magnet for different capacitor voltages, time $\mathrm{t}=2.6 \mathrm{~ms}$.

Figure 9 shows the magnetic induction in the middle of the Nd-Fe-B permanent magnet for different supply voltages of the magnetizer. This curve is nonlinear to capacitor voltage $2600 \mathrm{~V}$ and above this value the magnetic induction increases linearly.

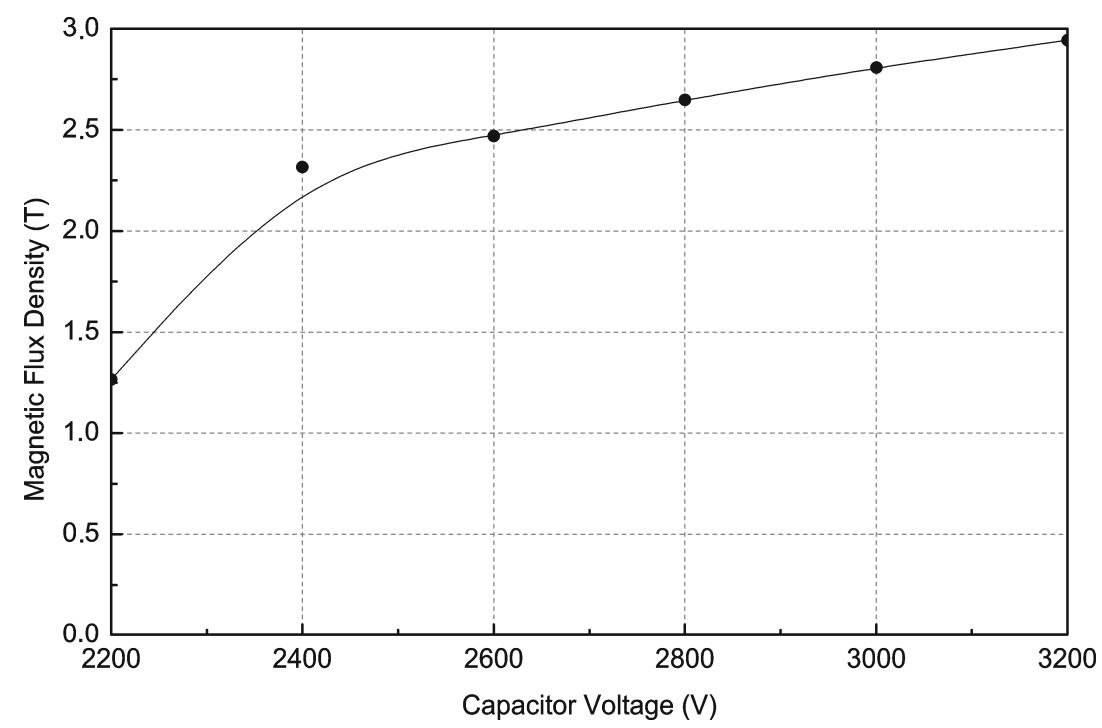

Figure 9. FEM simulations of magnetic induction in the centre of an Nd-Fe-B magnet as a function of capacitor voltages. 
The computer simulations presented in Figure 9 show that for voltage $U=2800 \mathrm{~V}$, the magnetic induction inside the magnet $\mathrm{B}=2.65 \mathrm{~T}$, which is practically sufficient for magnetization to saturation of an Nd-Fe-B type N38 permanent magnet.

Figure 10 shows the magnetic flux density in the magnetizing fixture and in the magnet during impulse magnetization. For this voltage, the magnetic flux density on the edge of the magnet is equal to $3.10 \mathrm{~T}$. We can observe the influence of eddy currents on the magnetic flux density distribution in the magnet.
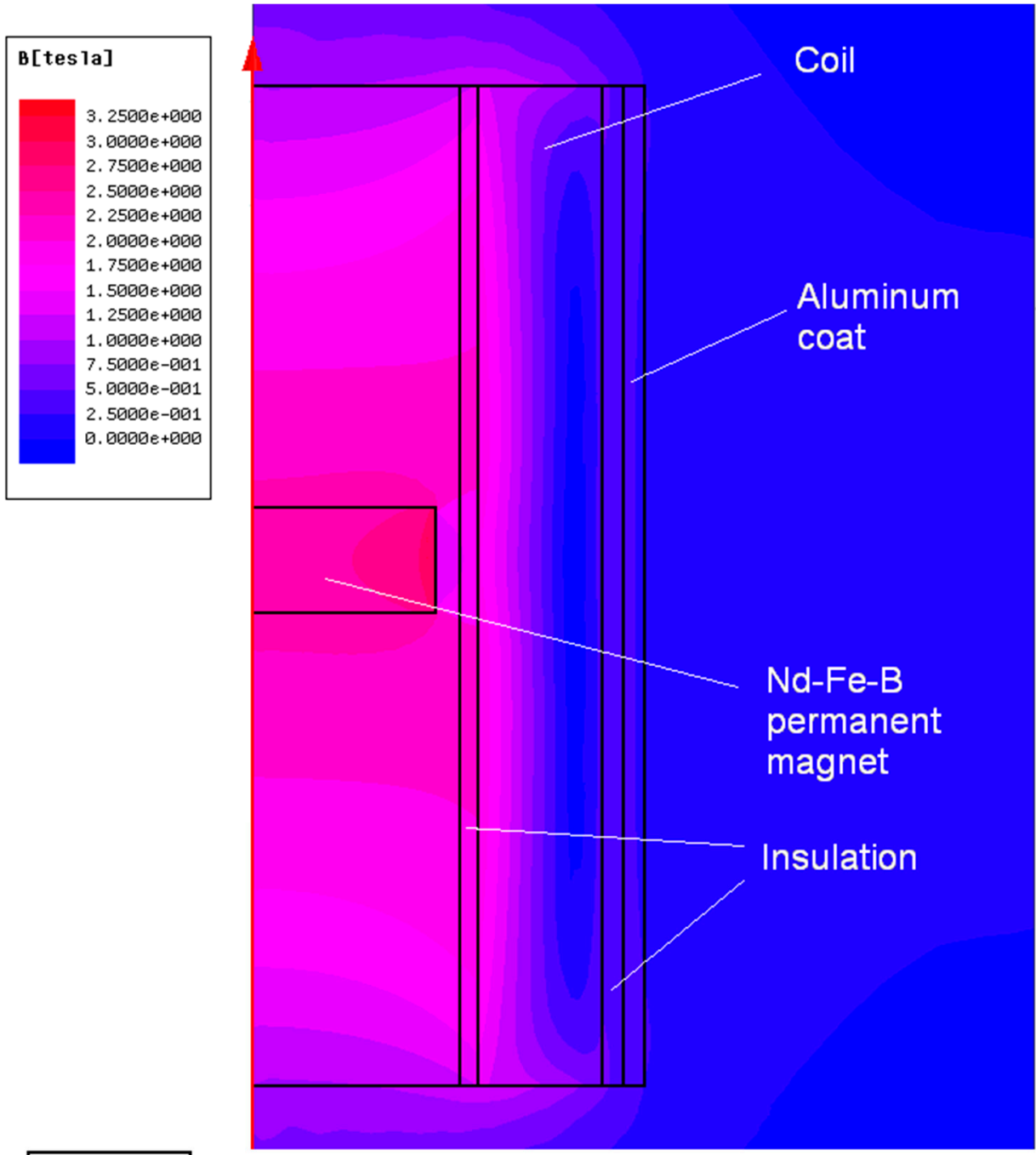

0

$100 \mathrm{~mm}$

Figure 10. FEM simulations in magnetizing fixture and Nd-Fe-B magnet for $U_{c}=2800 \mathrm{~V}, \mathrm{I}_{\mathrm{m}}=1032 \mathrm{~A}$, $\mathrm{t}=2.6 \mathrm{~ms}$.

\subsection{Measurements of Magnetic Induction on the Surface of the Magnet}

Measurements of induction on the surface of the cylindrical magnet $(70 \mathrm{~mm}$ diameter, $20 \mathrm{~mm}$ height) in the middle of the magnet and $5 \mathrm{~mm}$ from the edge of the magnet made of Nd-Fe-B material are shown in Figure 11 for different capacitor voltages. Measurements were conducted using a model 5070 teslameter made by F.W. Bell (Orlando, FL, USA), equipped with an SAH57-1904 axial probe. These curves also show that the capacitor voltage $U_{c}=2800 \mathrm{~V}$ is practically sufficient for magnetization of the magnet to saturation. 


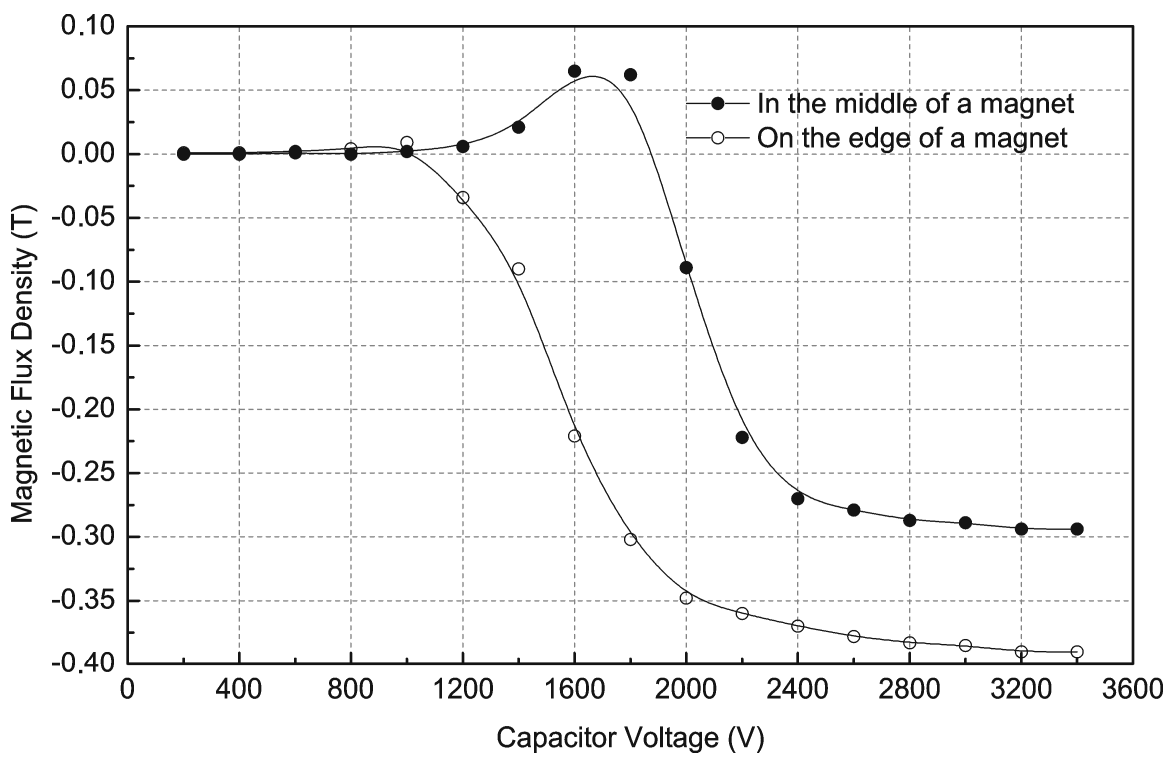

Figure 11. Measurements of magnetic induction on the surface of the magnet, for different capacitor voltages.

Photos were also taken of the magnetic field on the surface of the Nd-Fe-B permanent magnet. Figure 12 shows photos of the magnetic poles of the magnet displayed on a magnetic field viewing film - a special foil with nickel particles suspended in oil for observation of magnetic poles. In Figure 12a it is possible to see two poles of magnetic field, the inner bright circle dividing the magnet on two opposite poles. The values of the magnetic induction can be taken from Figure 6 for $\mathrm{U}_{\mathrm{c}}=1600 \mathrm{~V}$.

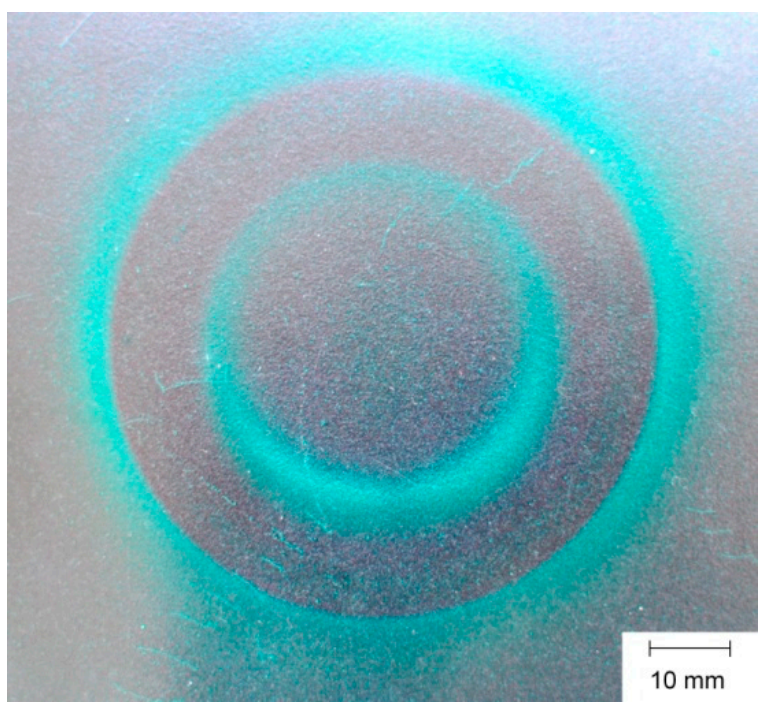

(a)

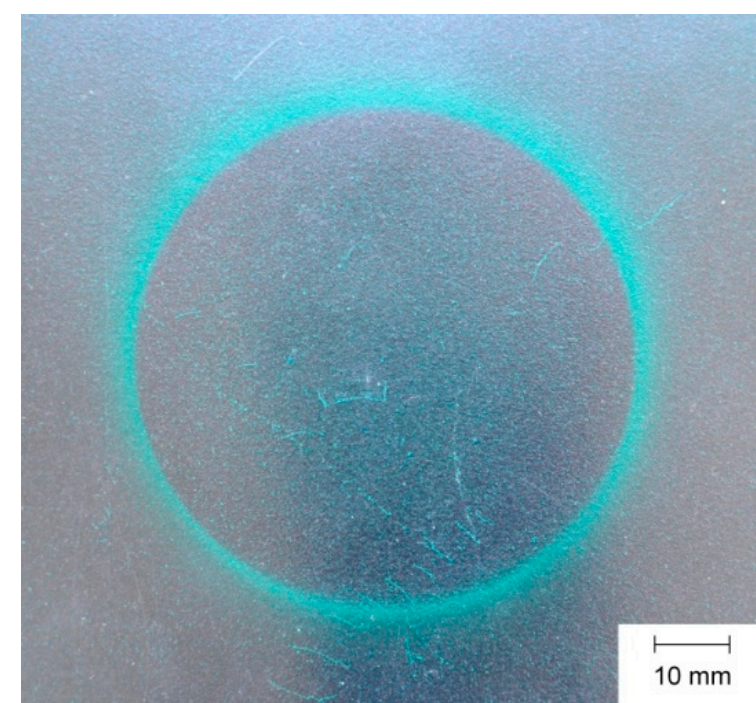

(b)

Figure 12. Photos of magnetic poles on the surface of N38 type Nd-Fe-B magnet for magnetization voltages (a) for $1600 \mathrm{~V}$ and (b) for $2800 \mathrm{~V}$.

\section{Conclusions}

Measurements of magnetic induction on the surface of the magnet show that for the applied voltage of $U_{c}=2800 \mathrm{~V}$ in the magnetizer to the capacitors, the magnet is practically saturated. Higher voltages do not increase the saturation of the magnet, but rather just cause higher energy 
costs, and a longer process of magnetization due to energizing of the capacitors and higher heating of the magnetization coil. Measurements confirming the numerical calculations and parameters of the electrical supply are determined optimally. The described method can be useful for producers of permanent magnets in the design and production of magnets with different shapes and dimensions. This method can also be useful for determining the magnetizing parameters of new types of permanent magnets, especially with low resistivity and high coercivity, when magnetized with very fast impulses of magnetic field.

Author Contributions: Marek Przybylski contributed the idea of the analysis of impulse the magnetization of Nd-Fe-B permanent magnets, Dariusz Kapelski measured the impulse currents and magnetic field, Barbara Ślusarek and Slawomir Wiak analysed the data and they critically reviewed the paper.

Conflicts of Interest: The authors declare no conflict of interest.

\section{References}

1. Ripka, P. Magnetic Sensors and Magnetometers; Artech House: Norwood, MA, USA, 2001.

2. Tumański, S. Handbook of Magnetic Measurements; CRC Press Taylor \& Francis: Boca Raton, FL, USA, 2011.

3. Zhao, B.; Wang, L.; Tan, J.-B. Design and Realization of a Three Degrees of Freedom Displacement Measurement System Composed of Hall Sensors Based on Magnetic Field Fitting by an Elliptic Function. Sensors 2015, 15, 22530-22546. [CrossRef] [PubMed]

4. Pacheco, C.J.; Bruno, A.C. A Noncontact Force Sensor Based on a Fiber Bragg Grating and its Application for Corrosion Measurement. Sensors 2013, 13, 11476-11489. [CrossRef] [PubMed]

5. Dubovikova, N.; Koleshnikov, Y.; Karcher, C. Experimental study of an electromagnetic flow meter for liquid metals based on torque measurement during pumping process. Meas. Sci. Technol. 2015, 26. [CrossRef]

6. Stupak, J.J., Jr. Methods of Magnetizing Permanent Magnets. Available online: http://oersted.com/ magnetizing.PDF (accessed on 21 April 2016).

7. Braybrooks, K.A. Improvements in and Relating to Magnetising Apparatus. British Patent 710738, 16 June 1954.

8. Zhilichev, Y.; Campbell, P.; Miller, D. In Situ Magnetization of Isotropic Permanent Magnets. In Proceedings of the IEEE Transactions on Magnetics, Amsterdam, the Netherlands, 28 April-2 May 2002; Volume 38, pp. 2988-2990.

9. Hsieh, M.-F.; Hsu, Y.-C. Characteristics Regulation for Manufacture of Permanent-Magnet Motors Using Post-Assembly Magnetization. IEEE Trans. Magn. 2007, 43, 2510-2512. [CrossRef]

10. Riley, C.D.; Jewell, G.W.; Howe, D. Design of Impulse Magnetizing Fixtures for the Radial Homopolar Magnetization of Isotropic NdFeB Ring Magnets. IEEE Trans. Magn. 2000, 36, 3846-3857. [CrossRef]

11. Nakata, T.; Takahashi, N. Numerical Analysis of Transient Magnetic Field in a Capacitor-Discharge Impulse Magnetizer. IEEE Trans. Magn. 1986, 22, 526-528. [CrossRef]

12. Jewell, G.W.; Howe, D.; Birch, T.S. Simulation of Capacitor Discharge Magnetisation. IEEE Trans. Magn. 1990, 26, 1638-1640. [CrossRef]

13. Jewell, G.W.; Howe, D. Post-Assembly Impulse Magnetization of Brushless DC Motors Equipped with Rare-Earth Magnets. In Proceedings of the 5th International Conference on Electrical Machines and Drives, London, UK, 11-13 September 1991; pp. 16-20.

14. Jewell, G.W.; Howe, D. Computer-Aided Design of Magnetizing Fixtures for the Post-Assembly Magnetization of Rare-Earth Permanent Magnet Brushless DC Motors. IEEE Trans. Magn. 1992, 28, 3036-3038. [CrossRef]

15. Lee, J.K. The Analysis of a magnetizing Fixture for a Multipole Nd-Fe-B Magnet. IEEE Trans. Magn. 1988, 24, 2166-2171. [CrossRef]

16. Ślusarek, B.; Gawryś, P.; Przybylski, M. Multi-pole magnetization of magnets of high density magnetic energy. In Proceedings of the 43th International Symposium of Electrical Machines, Poznań, Poland, 2-5 July 2007; pp. 87-90.

17. Riley, C.D.; Jewell, G.W.; Howe, D. The design and analysis of axial field multipole impulse magnetizing fixtures. J. Appl. Phys. 1998, 83, 7112-7114. [CrossRef] 
18. VanderHeiden, R.H.; Arkadan, A.A.; Brauer, J.R. Nonlinear Transient Finite Element Modeling of a Capacitor-Discharge Magnetizing Fixture. IEEE Trans. Magn. 1993, 29, 2051-2054. [CrossRef]

19. Jewell, G.W.; Howe, D.; Riley, C.D. The Design of Radial-Field Multipole Impulse Magnetizing Fixtures for Isotropic NdFeB Magnets. IEEE Trans. Magn. 1997, 33, 708-722. [CrossRef]

20. Ślusarek, B.; Gawryś, P.; Przybylski, M.; Maciejewski, G. Production method of magnetizing head for Multipolar magnetizing of durable magnets, especially of high magnetic density. Polish Patent 210617, 29 February 2012.

21. Karbowiak, M.; Kapelski, D.; Jankowski, M.; Przybylski, M.; Ślusarek, B. The Application of Multi-Pole Bonded Magnets in Electric Motors. In Zeszyty Problemowe-Maszyny Elektryczne; Branżowy Ośrodek Badawczo-Rozwojowy Maszyn Elektrycznych Komel: Katowice, Poland, 2011; pp. 49-52.

22. Karbowiak, M.; Kapelski, D.; Jankowski, M.; Przybylski, M.; Ślusarek, B. Multi-Pole Magnetization of Nd-Fe-B Bonded Magnets for Rotary Linear Actuators. In Elektronika; Wydawnictwo Czasopism i Książek Technicznych Sigma-Not: Warsaw, Poland, 2011.

23. Zhang, D.; Kim, H.-J.; Li, W.; Koh, C.-S. Analysis of Magnetizing Process of a New Anisotropic Bonded NdFeB Permanent Magnet Using FEM Combined with Jiles-Atherton Hysteresis Model. IEEE Trans. Magn. 2013, 49, 2221-2224. [CrossRef]

24. N38 Sintered Neodymium-Iron-Boron Magnets. Available online: http://www.magnesy.pl (accessed on 13 April 2016).

25. Przybylski, M.; Jankowski, B.; Kapelski, D.; Ślusarek, B. Measurements of impulse magnetic fields. Prz. Elektrotech. 2015, 48-50. [CrossRef]

(C) 2016 by the authors; licensee MDPI, Basel, Switzerland. This article is an open access article distributed under the terms and conditions of the Creative Commons Attribution (CC-BY) license (http://creativecommons.org/licenses/by/4.0/). 